\title{
Optical Design of the EPIC-IM Crossed Dragone Telescope
}

\author{
Huan Tran*a , Brad Johnson ${ }^{\mathrm{a}}$, Mark Dragovan ${ }^{\mathrm{b}}$, James Bock**b,c ${ }^{*}$, Abdullah Aljabri ${ }^{\mathrm{b}}$, Alex Amblard ${ }^{\mathrm{d}}$, \\ Daniel Bauman $^{\mathrm{e}}$, Marc Betoule ${ }^{\mathrm{f}}$, Talso Chui ${ }^{\mathrm{b}}$, Loris Colombo ${ }^{\mathrm{g}}$, Asantha Cooray ${ }^{\mathrm{d}}$, Dustin Crumb ${ }^{\mathrm{h}}$, \\ Peter Day $^{\mathrm{b}}$, Clive Dickenson ${ }^{\mathrm{c}, \mathrm{i}}$, Darren Dowell ${ }^{\mathrm{b}, \mathrm{c}}$, Sunil Golwala ${ }^{\mathrm{c}}$, Krzysztof Gorski ${ }^{\mathrm{b}, \mathrm{c}}$, Shaul \\ Hanany, Warren Holmes ${ }^{\mathrm{b}}$, Kent Irwin ${ }^{\mathrm{k}}$, Brian Keating, Chao-Lin Kuo ${ }^{\mathrm{m}}$, Adrian Lee ${ }^{\mathrm{a}}$, Andrew \\ Lange $^{\mathrm{c}, \mathrm{b}}$, Charles Lawrence ${ }^{\mathrm{b}}$, Steve Meyer ${ }^{\mathrm{n}}$, Nate Miller ${ }^{1}$, Hien Nguyen ${ }^{\mathrm{b}}$, Elena Pierpaolig, Nicolas \\ Ponthieu ${ }^{\mathrm{f}}$, Jean-Loup Puget ${ }^{\mathrm{f}}$, Jeff Raab ${ }^{\mathrm{o}}$, Paul Richards ${ }^{\mathrm{a}}$, Celeste Satter ${ }^{\mathrm{b}}$, Mike Seiffert ${ }^{\mathrm{b}}$, Meir \\ Shimon, Brett Williams ${ }^{\mathrm{b}}$, Jonas Zmuidzinas ${ }^{\mathrm{c}, \mathrm{b}}$ \\ ${ }^{a}$ University of California Berkeley, Berkeley, CA, 94720, USA; \\ ${ }^{\mathrm{b} J e t}$ Propulsion Laboratory, 4800 Oak Grove Dr., Pasadena, CA, 91109, USA; \\ ${ }^{\mathrm{c} C a l i f o r n i a}$ Institute of Technology, 1200 East California Blvd., Pasadena, CA, 91125, USA; \\ ${ }^{\mathrm{d}}$ University of California Irvine, Irvine, CA, 92697, USA; \\ ${ }^{\mathrm{e}}$ Harvard University, Cambridge, MA, 02138, USA; \\ Institute d'Astrophysique Spatial, 91405 Orsay Cedex, France; \\ ${ }^{g}$ University of Southern California, Los Angeles, CA, 90089, USA; \\ ${ }^{\mathrm{h}}$ ATK Aerospace Systems, 404 N. Halstead St., Pasadena, CA, 91107, USA; \\ ${ }^{\mathrm{i} J o d r e l l ~ B a n k}$ Centre for Astrophysics, U. Manchester, Oxford Road, Manchester, M13 9PL, UK; \\ ${ }^{\mathrm{j}}$ University of Minnesota, Minneapolis, MN, 55455, USA; \\ ${ }^{\mathrm{k}}$ National Institute of Standard, 325 Broadway, Boulder, CO, 80305, USA; \\ ${ }^{1}$ University of California San Diego, 9500 Gilman Dr., La Jolla, CA, 92093, USA; \\ ${ }^{\mathrm{m}}$ Stanford University, Stanford, CA, 94305, USA; \\ ${ }^{\mathrm{n}}$ University of Chicago, 5801 South Ellis Ave., Chicago, IL, 60637, USA; \\ ${ }^{\circ}$ Northrup Grumman Aerospace Systems, 1 Space Park, Redondo Beach, CA, 90278, USA;
}

\begin{abstract}
The Experimental Probe of Inflationary Cosmology - Intermediate Mission (EPIC-IM) is a concept for the NASA Einstein Inflation Probe satellite. EPIC-IM is designed to characterize the polarization properties of the Cosmic Microwave Background to search for the B-mode polarization signal characteristic of gravitational waves generated during the epoch of Inflation in the early universe. EPIC-IM employs a large focal plane with 11,000 detectors operating in 9 wavelength bands to provide 30 times higher sensitivity than the currently operating Planck satellite. The optical design is based on a wide-field $1.4 \mathrm{~m}$ crossed-Dragone telescope, an aperture that allows not only comprehensive measurements of Inflationary B-mode polarization, but also measurements of the E-mode and lensing polarization signals to cosmological limits, as well as all-sky maps of Galactic polarization with unmatched sensitivity and angular resolution. The optics are critical to measuring these extremely faint polarization signals, and any design must meet demanding requirements on systematic error control. We describe the EPIC-IM crossed Dragone optical design, its polarization properties, and far-sidelobe response.
\end{abstract}

Keywords: Cosmic microwave background, cosmology, inflation, polarization, optical design, satellite, mission concept

\footnotetext{
*This paper honors the memory of Huan Tran, and his dedicated contributions to the EPIC-IM optical design. Sections 2-7 are taken from the chapter he wrote with Brad Johnson and Mark Dragovan for the EPIC-IM report [2]. Huan is greatly missed by his friends and colleagues.
}

**jjb@astro.caltech.edu; phone 1626 395-2017; fax 1626 395-2366

Space Telescopes and Instrumentation 2010: Optical, Infrared, and Millimeter Wave, edited by Jacobus M. Oschmann Jr., Mark C. Clampin, Howard A. MacEwen, Proc. of SPIE Vol. 7731, 77311R - () 2010 SPIE · CCC code: 0277-786X/10/\$18 · doi: 10.1117/12.857423 


\section{INTRODUCTION}

The theory of inflation, inspired in part by the extreme isotropy of the CMB, is now a cornerstone of modern cosmology. Inflation posits that an exponential expansion stretched space in the first moments after the Big Bang and promoted microscopic quantum fluctuations to perturbations on cosmological scales. The theory has passed a series of rigorous experimental tests, driven by increasingly precise measurements of the CMB anisotropy, both in temperature and polarization. Predictions of inflation have now been verified in the scale-invariant spectrum on large angular scales, the nearly perfectly flat geometry from the apparent angular scale of the acoustic peaks in the spectrum, the adiabatic and Gaussian nature of anisotropies, and the anti-correlation of temperature and polarization on scales outside of the causal horizon. While evidence for inflation now seems inescapable, we still do not understand the physical mechanism or energy scale behind inflation.

Inflation theory generally predicts a stochastic background of gravitational waves - ripples in space-time that travel at the speed of light. The amplitude and the shape of the gravitational wave spectrum contain unique information about the physics of the early Universe that is not captured by the observed density perturbations. A detection of the primordial gravitational wave background would be a truly spectacular achievement and will not only establish inflation as the source of cosmological perturbations, but also allow a way to connect inflationary models to fundamental physics at a specific energy scale [1].

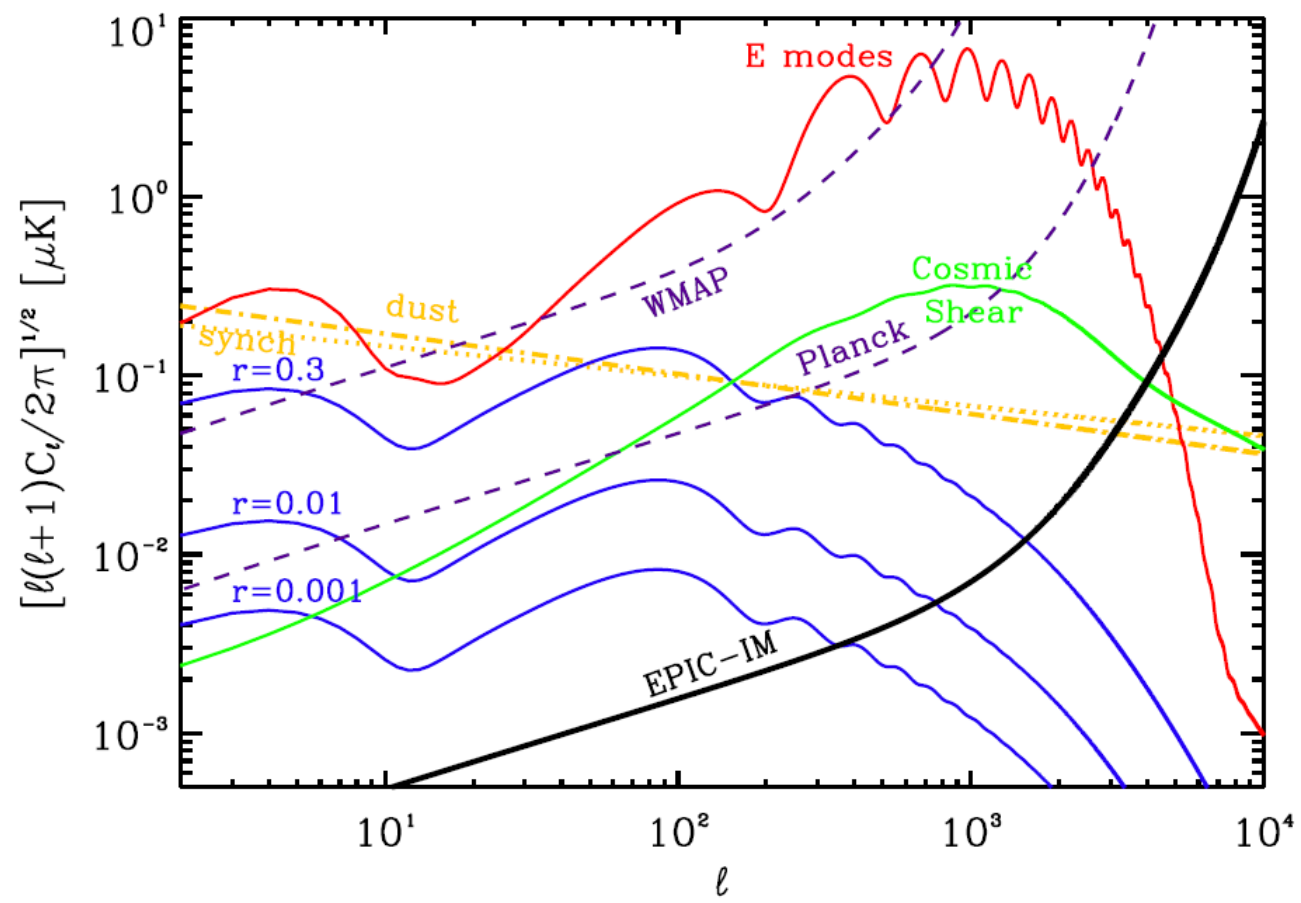

Figure 1. The sensitivity of EPIC-IM, WMAP and Planck to CMB polarization anisotropy. E-mode polarization from scalar perturbations is shown in red; B-mode polarization from tensor perturbations is shown in blue for $\mathrm{r}=$ 0.3 and $\mathrm{r}=0.01$; and B-mode polarization produced by lensing of the E-mode polarization is shown in green. The science goal of EPIC is to measure the inflationary B-mode spectrum the level of $r=0.01$ for the entire $2<\ell<$ 200 multipole range after foreground subtraction. Expected B-mode foreground power spectra for polarized dust (orange dash-dotted) and synchrotron (orange dotted) at $70 \mathrm{GHz}$. The band-combined instrumental sensitivity of EPIC is given assuming the $4 \mathrm{~K}$ telescope option and 4 years of observations. WMAP assumes an 8-year mission life; Planck assumes 2.5 years at HFI flight sensitivity. Note that the sensitivity curves show band-combined sensitivities to $C_{\ell}$ in broad $\Delta \ell / \ell=0.3$ bins in order to compare the full raw statistical power of the three experiments in the same manner. The final sensitivity to $r$ after foreground removal will naturally be reduced.

The Experimental Probe of Inflationary Cosmology (EPIC) [2] will pursue the CMB polarization signature associated with the inflationary gravitational wave signal. The EPIC-IM (Intermediate Mission) configuration contrasts with a previous low-resolution EPIC-LC configuration based on 6 small $30 \mathrm{~cm}$ telescopes [3]. EPIC-IM with a $1.4 \mathrm{~m}$ aperture 
provides the necessary angular resolution to measure the lensing and scalar polarization signals shown in Fig.1. EPICIM has sufficient sensitivity, frequency coverage, and control of systematic errors [4] to make a precise measurement of the inflationary B-mode spectrum to astrophysical limits, likely set by the removal of Galactic foregrounds. In addition, EPIC-IM has the arc-minute angular resolution needed to study lensing polarization, enabling precise determination of neutrino masses and late dark energy. EPIC-IM will produce maps of the gravitational potential useful in studies of large scale structure in conjunction with e.g. galaxy surveys, weak lensing, and baryon acoustic oscillations. EPIC will extract all the information contained in $\mathrm{CMB}$ polarization about the history of reionization, and has high-frequency bands devoted to study dust polarization and its relationship to Galactic magnetic fields.

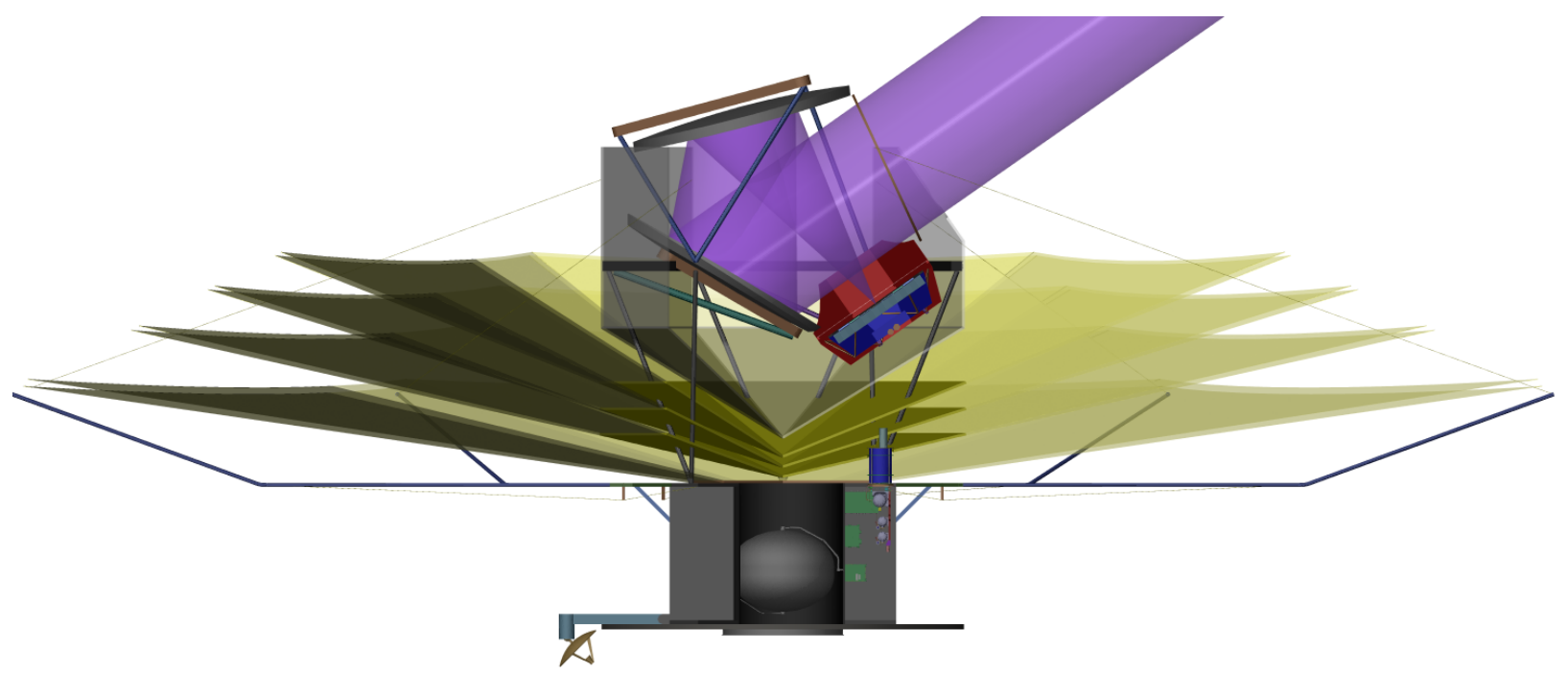

Figure 2. Overview of the EPIC-IM satellite. The instrument is based on a large $100 \mathrm{mK}$ focal plane at the focus of a 1.4 crossed-Dragone telescope. The telescope and aperture stop are cooled to $4 \mathrm{~K}$, and the optical enclosure to $18 \mathrm{~K}$, by a mechanical refrigeration system. A 4-stage passive cooling system reduces parasitic power on the mechanical cooler. A 4-stage sunshield enables a spinning and precessing scan strategy which is optimized for polarization measurements from EPIC's Sun-Earth L2 Lagrange halo orbit. Solar power and telemetry downlink are located on the Sun/Earth-facing side of the spacecraft at bottom.

EPIC-IM employs a large $100 \mathrm{mK}$ focal plane with 11,000 detectors operating in 9 frequency bands. EPIC-IM has unprecedented system sensitivity, a factor of 30 improvement over Planck. The detectors operate in matched pairs in a single band, with one detector sensing vertical polarization and one sensing horizontal polarization. The orientation of these pairs is varied over the focal plane to provide uniform instantaneous sampling of $\mathrm{Q}$ and $\mathrm{U}$. The detectors are mounted in hexagonal tiles, packed with the highest frequencies located at the center of the focal plane where aberrations are lowest. The detector field of view must be collimated at the focal plane, either by antennas or feeds.

The telescope is cooled by a combination of passive cooling and a mechanical $4 \mathrm{~K}$ cryocooler [5], and the focal plane is cooled to $100 \mathrm{mK}$ by either an adiabatic demagnetization refrigerator or a closed-cycle dilution refrigerator [6]. The passive cooling system uses a 3-stage V-groove radiator that cools to a minimum temperature of $\sim 30 \mathrm{~K}$. The instrument is mounted to the spacecraft on a low thermal conductivity bipod, with each V-groove radiator mounted to the bipod in sequence. The optics are enclosed in a light-weight optics shield, and the shield is actively cooled to $18 \mathrm{~K}$ by an expansion stage of the $4 \mathrm{~K}$ cryocooler. The sub- $\mathrm{K}$ cooler operates from $4 \mathrm{~K}$ to provide continuous cooling at $100 \mathrm{mK}$, with an intermediate stage at $\sim 1 \mathrm{~K}$ to reduce parasitic conductive and optical loads. A large deployed 4-stage sunshield keeps radiation from the sun, earth and moon from reaching the instrument or optics. Power is provided by rigidly mounted solar panels attached to the bottom of the spacecraft bus. A deployed gimbaled Ka-band antenna is used for data downlink.

EPIC-IM conducts science observations from an earth-sun L2 halo orbit. EPIC-IM uses a spinning and precessing scan strategy, rotating about the spin axis at $\sim 0.5 \mathrm{rpm}$ while precessing about the sun-line every hour. This strategy provides coverage patterns on the sky that are ideal for a polarization measurement, while keeping the solar illumination on the sunshield and solar panels constant. The scan strategy produces annual maps with a high degree of rotation of the 
instrument view angle on any given region of sky. It also produces daily maps covering more than half the sky with excellent angular coverage over most of this region. These maps can be used to track a variety of systematics effects on multiple timescales, and to form multiple difference maps for monitoring systematic errors. In order to perform telemetry communications during observations, the downlink antenna is gimbaled to maintain a constant line of contact to Earth.

Table 1 Summary of the EPIC-IM Mission

\begin{tabular}{|l|l||l|l|}
\hline Optics & $1.4 \mathrm{~m}$ wide-field crossed Dragone & Total Delta-V & $170 \mathrm{~m} / \mathrm{s}$ \\
\hline Orbit & Sun-Earth L2 Halo & Payload Power & $440 \mathrm{~W}(\mathrm{CBE})$ \\
\hline Mission Life & 4 years & Spacecraft Power & $530 \mathrm{~W}(\mathrm{CBE})$ \\
\hline Launch Vehicle & Atlas V 401 & Total Power & $1392 \mathrm{~W}(\mathrm{w} 43 \%$ cont.) \\
\hline \# Detectors & $11094 \mathrm{TES}$ or MKID & Payload Mass & $813 \mathrm{~kg}(\mathrm{CBE})$ \\
\hline Bands & $30 / 45 / 70 / 100 / 150 / 220 / 340 / 500 / 850 \mathrm{GHz}$ & Spacecraft Mass & $584 \mathrm{~kg}(\mathrm{CBE})$ \\
\hline Sensitivity & $0.9 \mathrm{uK}$ arcmin; $\sim 1000$ Planck missions & Total Mass & $2294 \mathrm{~kg}(\mathrm{w} / 43 \%$ cont.) \\
\hline Spacecraft & 3-axis commercial & Mass Margin & $1287 \mathrm{~kg}(36 \%)$ \\
\hline Data Rate & 7.7 Mbps & Cost & $\$ 920 \mathrm{M}(\mathrm{FY} 09)$ \\
\hline
\end{tabular}

EPIC-IM uses an off-axis crossed-Dragone telescope that provides a very large unabberated field of view with low polarization artifacts. The design provides a flat and telecentric focal plane, so that all the focal plane feeds look straight up into the telescope over the field of view. A $1.4 \mathrm{~m}$ cooled absorbing stop sets the effective projected aperture, and the primary and secondary mirrors are oversized to minimize spillover. This design avoids refracting optics, and is thus ideal for a single large multi-color focal plane. Far-sidelobe response is controlled by a combination of underilluminating the mirrors, and using absorbing baffles at the primary, secondary, and aperture stop. These baffles must be appropriately cold and temperature stable so as to not introduce appreciable photon noise and signal drifts. The design must meet demanding systematic requirements on main effects and far-sidelobes, quantified in [2], which can otherwise imprint false polarization signals.

\section{CROSSED DRAGONE TELESCOPE}

For the EPIC-Intermediate Mission, we have chosen to study a dual reflector antenna system coupled directly to the focal plane without reimaging optics. The particular mirror configuration chosen is the Crossed Dragone, also known as the Side-Fed, or Compact Range Antenna. The motivation for choosing this configuration is discussed in [7]. To summarize, the Crossed Dragone provides a very large FOV and is compatible with a flat, telecentric focal plane without the need for refractive reimaging optics. This simple feature avoids many of the issues with refracting elements such as AR coatings, index uncertainty and dispersion, birefringence and surface roughness.

The specific design considerations for EPIC IM are:

1. Compact size compatible with the launch shroud

2. Boresight angle to match $55^{\circ}$ angle in scan strategy

3. Large FOV

4. Cold aperture stop

5. Telecentric focal plane

6. Beam scale polarization distortions

7. Low far sidelobe level to minimize Galactic contamination

8. Weight

9. Cooling

The mirrors are fabricated by either lightweight carbon fiber or silicon carbide with aluminized coatings. Cooling considerations dictate that the focal plane be placed close to the spacecraft bus, and that the optical system be surrounded by an optics box to reduce thermal radiation from warmer stages. With this design choice, much of the optical design can be evaluated using geometric optics, with the polarized beams and sidelobes requiring more involved simulations. 
By forcing the focal plane to be rigorously telecentric, we have moved the aperture stop from the primary to a location in front of the primary, far enough from the beam so that a cold physical aperture can be used to intercept sidelobes from the focal plane pixels.

Table 2 Parameters used to specify the EPIC-IM Crossed Dragone

\begin{tabular}{|l|l|l|}
\hline Parameter & Value & Description \\
\hline Effective focal length & $3.0 \mathrm{~m}$ & Sets the plate scale at the Gregorian focus \\
\hline Primary focal length & $5.6 \mathrm{~m}$ & Focal length of the paraboloidal primary \\
\hline Primary offset & $5.11 \mathrm{~m}$ & $\begin{array}{l}\text { Displacement of the optical axis from the symmetry axis of the } \\
\text { primary, in other words, the distance that the primary is off center }\end{array}$ \\
\hline Secondary semi focal spacing & $3.55 \mathrm{~m}$ & Half the separation of the foci of the ellipsoidal secondary \\
\hline Horn divergence & $13.13 \mathrm{deg}$ & $\begin{array}{l}\text { Half the opening angle at the focal plane, defining the aperture and } \\
\text { the marginal rays }\end{array}$ \\
\hline
\end{tabular}

Table 3 Optical Parameters for the EPIC-IM Optical system

\begin{tabular}{|l|l|l|}
\hline Parameter & Value & Description \\
\hline Effective Focal Length & $3.0 \mathrm{~m}$ & Focal length of mirror system, also used to calculate plate scale \\
\hline Effective Aperture & $1.4 \mathrm{~m}$ & Defined by entrance aperture \\
\hline F/\# & 2.14 & \\
\hline FOV X & $30.3 \mathrm{deg}$ & Available FOV in long dimension at $30 \mathrm{GHz}$ \\
\hline FOV Y & $19.9 \mathrm{deg}$ & Available FOV in short dimension at $30 \mathrm{GHz}$ \\
\hline
\end{tabular}

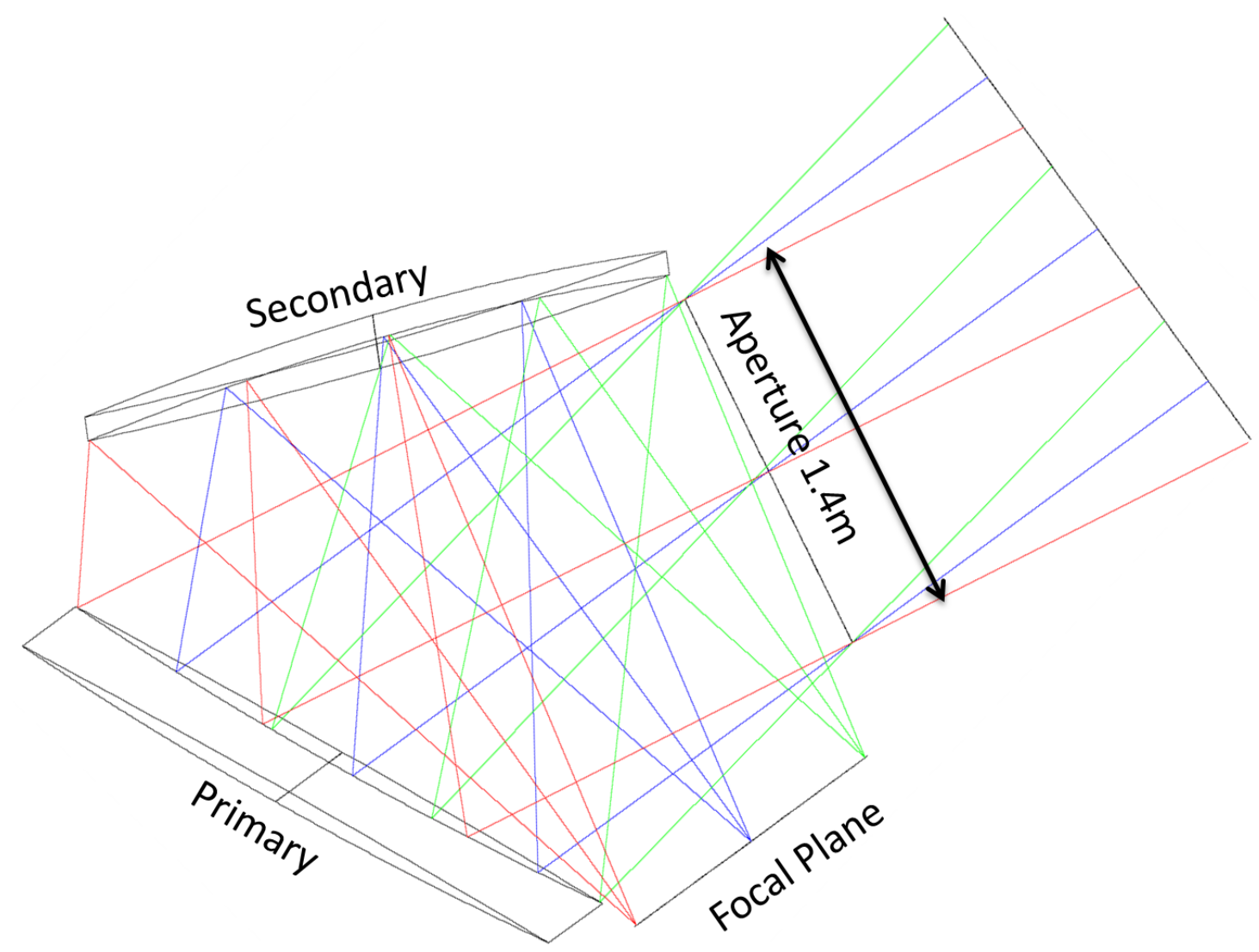

Figure 3. Raytrace diagram of EPIC-IM. The four main components are shown, along with rays from the extreme fields. The marginal rays in this figure represent the $-10 \mathrm{~dB}$ tapered rays from each pixel for the $2 \mathrm{f} \lambda$ case. For the $3.25 \mathrm{f} \lambda$ case, the marginal rays are the $-25 \mathrm{~dB}$ tapered rays. The focal plane and mirror projection here shows the short dimension. The focal plane and mirrors are longer into the plane of the figure. 
Given that the EPIC-IM design also does not include any refracting elements, the polarization performance of the mirrors can be accurately simulated using physical optics. The Dragone condition for a dual reflector pair produces a central feed with very low cross-polarization. The condition at the same time produces the very large FOV with a side effect of low polarization errors. It is likely that the polarization performance of the optical system will be limited by the focal plane coupling technology.

While the current EPIC-IM optical design was chosen to maximize throughput, several measures were undertaken to minimize and analyze the far sidelobes produced by diffraction from edges. The baffling plan incorporates an absorbing aperture stop, absorbing rings around the mirrors, and a reflective optics box. In addition to the baffling, the focal plane detectors under-illuminate the mirrors, with an edge taper that depends on the focal plane packing. The far-sidelobes are simulated using both intensive Physical Optics calculations and using the Geometric Theory of Diffraction. Finally, the Galactic contamination from the far-sidelobes is evaluated by convolving the simulated beams with a Galactic foreground model.

Table 4 Derived physical parameters used to construct the EPIC-IM Crossed Dragone

\begin{tabular}{|l|l|l|}
\hline Parameter & Value & Description \\
\hline Dragone Tilt & $61.821 \mathrm{deg}$ & Angle between symmetry axis of primary and secondary \\
\hline Feed Tilt & $28.179 \mathrm{deg}$ & Angle between axis of secondary and axis of the feed (normal to focal plane) \\
\hline Secondary Radius & $7.2214 \mathrm{~m}$ & Radius of Curvature of the Secondary, used in Zemax to specify surface \\
\hline Conic Constant & -5.9704 & Conic Constant of the Secondary \\
\hline Primary rim Y & $2.02 \mathrm{~m}$ & Projected Minor axis of primary aperture \\
\hline Primary Rim X & $2.34 \mathrm{~m}$ & Projected Major axis of primary aperture \\
\hline Secondary offset & $1.11 \mathrm{~m}$ & Offset of secondary rim from symmetry axis \\
\hline Secondary Rim Y & $2.2 \mathrm{~m}$ & Projected Minor axis of secondary aperture \\
\hline Secondary Rim X & $2.6 \mathrm{~m}$ & Projected Major axis of secondary aperture \\
\hline Aperture Diameter & $1.4 \mathrm{~m}$ & Entrance aperture is circular \\
\hline Aperture location & $3.10 \mathrm{~m}$ & $\begin{array}{l}\text { Distance between the center of the entrance aperture to the vertex of the } \\
\text { primary. In the modified Crossed Dragone, the aperture is not the primary. } \\
\text { Once the focal plane is forced to be telecentric, the aperture moves away } \\
\text { from the primary toward the far edge of the secondary. }\end{array}$ \\
\hline Aperture tilt & $10 \mathrm{deg}$ & $\begin{array}{l}\text { Tilt of the entrance aperture. The crossed dragone is highly decentered, and } \\
\text { the ideal entrance pupil is tilted. }\end{array}$ \\
\hline
\end{tabular}

\section{GEOMETRIC DESIGN AND ANALYSIS}

\subsection{Optical Layout}

It is well known that dual reflector mirrors pairs can be completely specified by five parameters [8]. The exact mirror parameters in this study were chosen to produce an ultra-compact mirror pair, with $15 \mathrm{~cm}$ clearance between the beam and the focal plane for filters. The location of the aperture stop was also tuned to be close to the secondary without interfering with the focal plane. This particular realization of the Crossed Dragone is a "local-minimum" in parameter space, and it is possible that a better realization can be found with a more exhaustive search.

The overall scale of the telescope is constrained by the desire to place the focal plane near the spacecraft bus, and at the same time orient the bore sight at $55^{\circ}$ from the spacecraft rotation axis. Setting the focal plane near the bus allows for more efficient cooling, and the bore sight requirement is set from the desired scan pattern. Applying the orientation constraint and leaving space for mirror supports and the hexagonal optics box allows a final aperture at $1.4 \mathrm{~m}$. The physical parameters for the EPIC-IM optical elements are listed in Tables $2-4$, and a geometric raytrace produced by Zemax is shown in Fig. 3.

It is important to note that the focal plane and mirrors have an elliptical outline to maximize throughput. The width of the focal plane in the short dimension is limited by vignetting, while the width in the long dimension is limited by geometric aberrations relative to $30 \mathrm{GHz}$ diffraction. The sizes of the mirrors in Fig. 3 were chosen such that the extreme fields were not vignetted by the mirror rims, leaving the cold aperture as the limiting stop. 


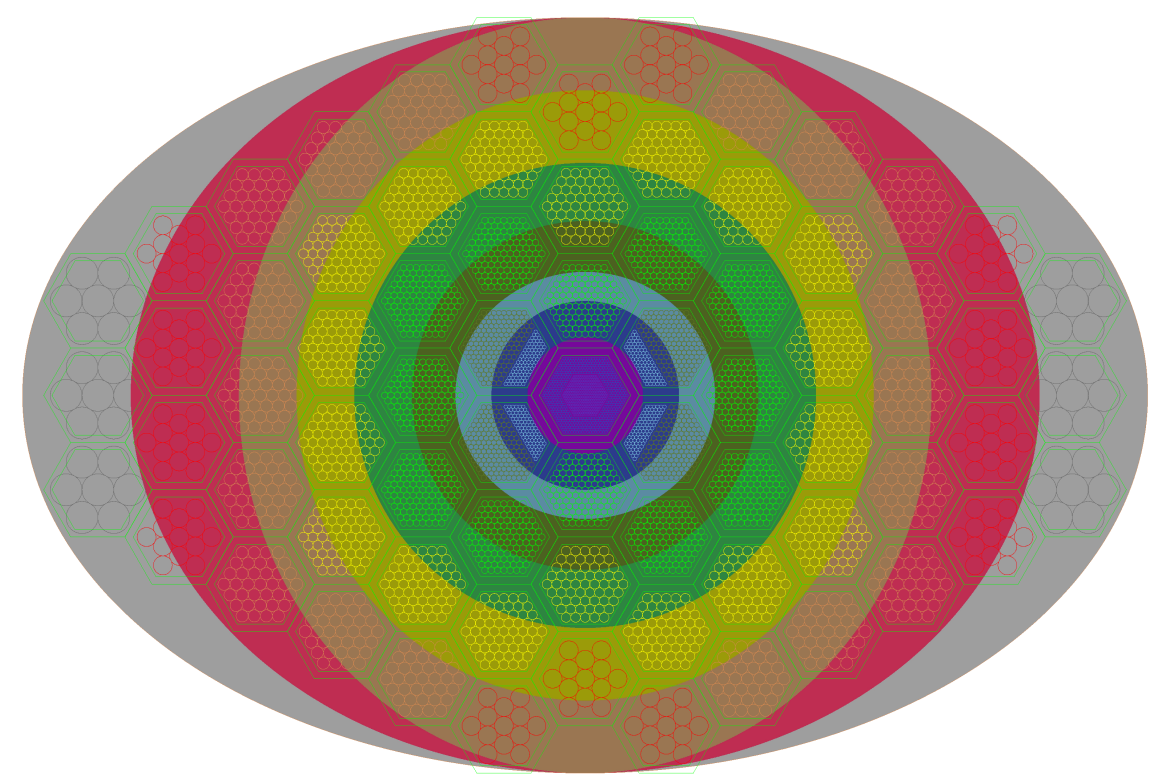

Figure 4. Available focal plane area as a function of position for the $4 \mathrm{~K}$ option. The colored regions denote the available area, and the outline of the wafer is overlaid. The extent of the shaded region is bounded by aberrations in the long dimension, defined by $\mathrm{Streh}=0.8$. The extant in the short dimension is set by vignetting. The colored bands (from inside to outside) are 850, 500, 350, 220, 150, 100, 70, 45 and $30 \mathrm{GHz}$.

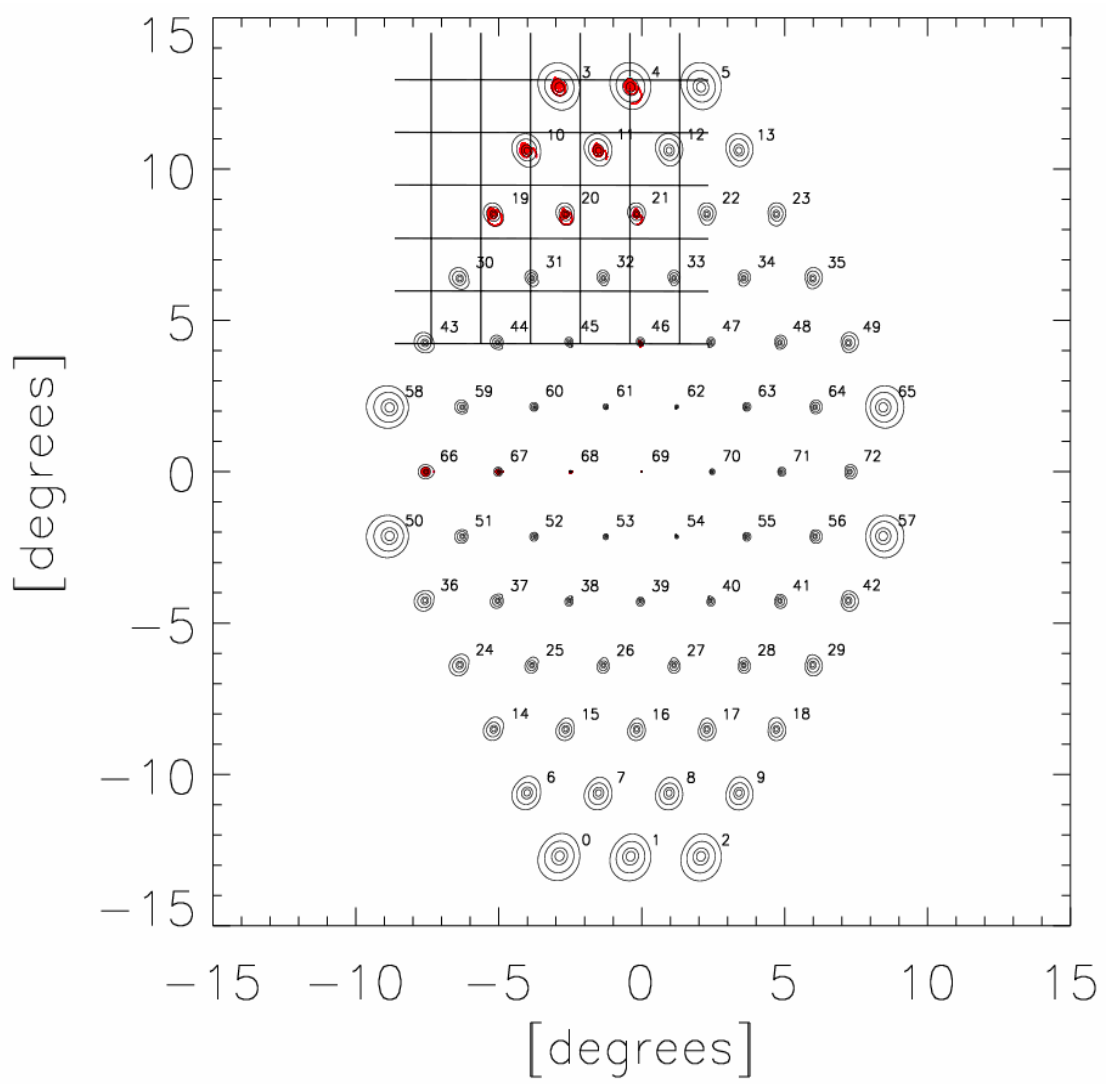

Figure 5. Geometric Optics vs Physical optics simulation of the beams. The contour plot is the co-polarized result of the Physical optics simulations. Each beam shown here is from a feed centered at each wafer shown in figure 6.2. The lowest contour shown is $-20 \mathrm{~dB}$. The spot diagram from ZEMAX for selected fields is overlaid in red. 


\subsection{Optical Layout}

The Crossed Dragone has very low geometric aberrations, leading to a very large field of view and throughput. The EPIC-IM cold mission concept takes advantage of this performance with a large focal plane, leading to high system sensitivity. There is enough aberration free area on the focal plane to accommodate 11,000 focal plane detectors in nine frequency bands. The central pixel in the focal plane is perfectly corrected, and aberrations grow slowly with radial feed position. The EPIC-IM focal plane takes advantage of this slow degradation by using the center of the focal plane for higher frequency feeds as shown in Fig. 4.
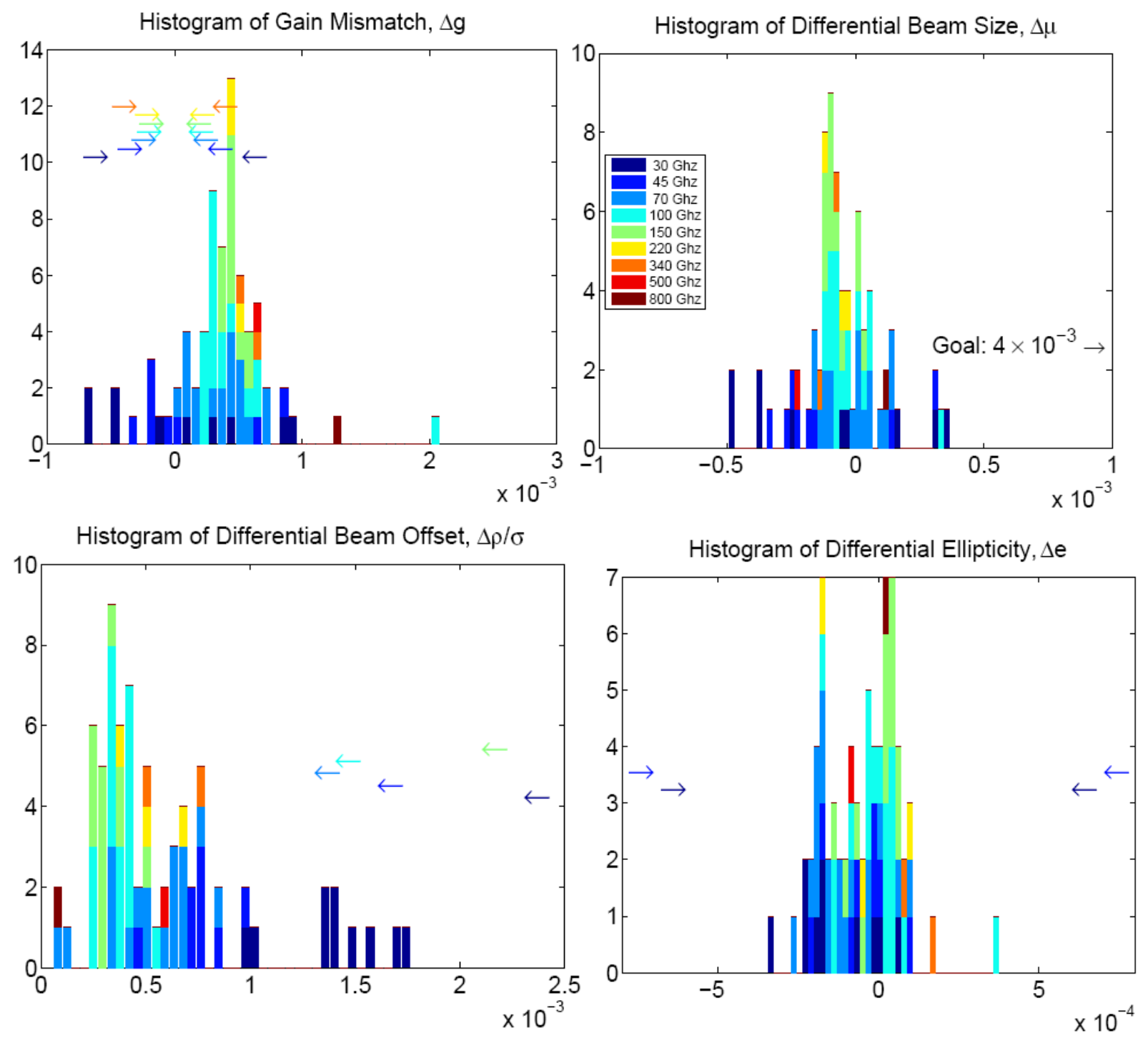

Figure 6. Histograms of main beam effects. Refer to Section 5.4 of the EPIC-IM report [2] for definitions of each effect. Histograms are color coded by frequency. Colored arrows denote the frequency dependant goals [2] for systematic error suppression. Goals for some frequencies are outside of plot area. Most simulated effects off the telescope are smaller than the goals, with the exception of the differential gain. The Gain mismatch is caused by the finite conductivity of the reflector material, and is degenerate with calibration between bolometer pairs, which will be removed by in-flight measurement. 


\section{MAIN BEAM EFFECTS}

The effects of diffraction on mirror based optical systems can be accurately simulated with physical optics. Without reimaging lenses, the accuracy of physical optics modeling of the EPIC-IM beam performance is limited only by knowledge of the focal plane coupling technology. For the purpose of evaluating the telescope performance, we have modeled the focal plane elements as ideal circular Gaussian tapered feeds.

The EPIC-IM optics were simulated using GRASP-9. One $2 \mathrm{f} \lambda$ feed at the appropriate wavelength was simulated at the center of each focal plane hex shown in Fig. 4. For the purpose of this simulation, it is assumed that a $2 \mathrm{f} \lambda$ feed spacing corresponds to an aperture beam taper of $-10 \mathrm{~dB}$. Both orthogonal linear polarizations were simulated to evaluate polarization artifacts. The mirrors were also assumed to have a finite conductivity of $2.5 \mathrm{e} 7 \mathrm{~S} / \mathrm{m}$, appropriate for aluminum, allowing us to simulate instrumental polarization.

\subsection{Geometrical Optics vs. Physical Optics}

Fig. 5 shows a comparison between the co-polarized output of the simulation and the geometric spot diagrams from raytracing. From the plot, it is clear that the PO simulation adequately reproduces the geometric aberrations, and furthermore that the frequency at each field locations was properly chosen to remain dominated by diffraction effects.

\subsection{Comparison with Systematics Requirements}

The results of the Grasp-9 main beam simulations can be evaluated for polarization effects induced by imperfect optical performance. Within a feed, the two orthogonally polarized beam patterns are fit to two-dimensional Gaussian models, and the resulting fit parameters are used to calculate the main beam effects.
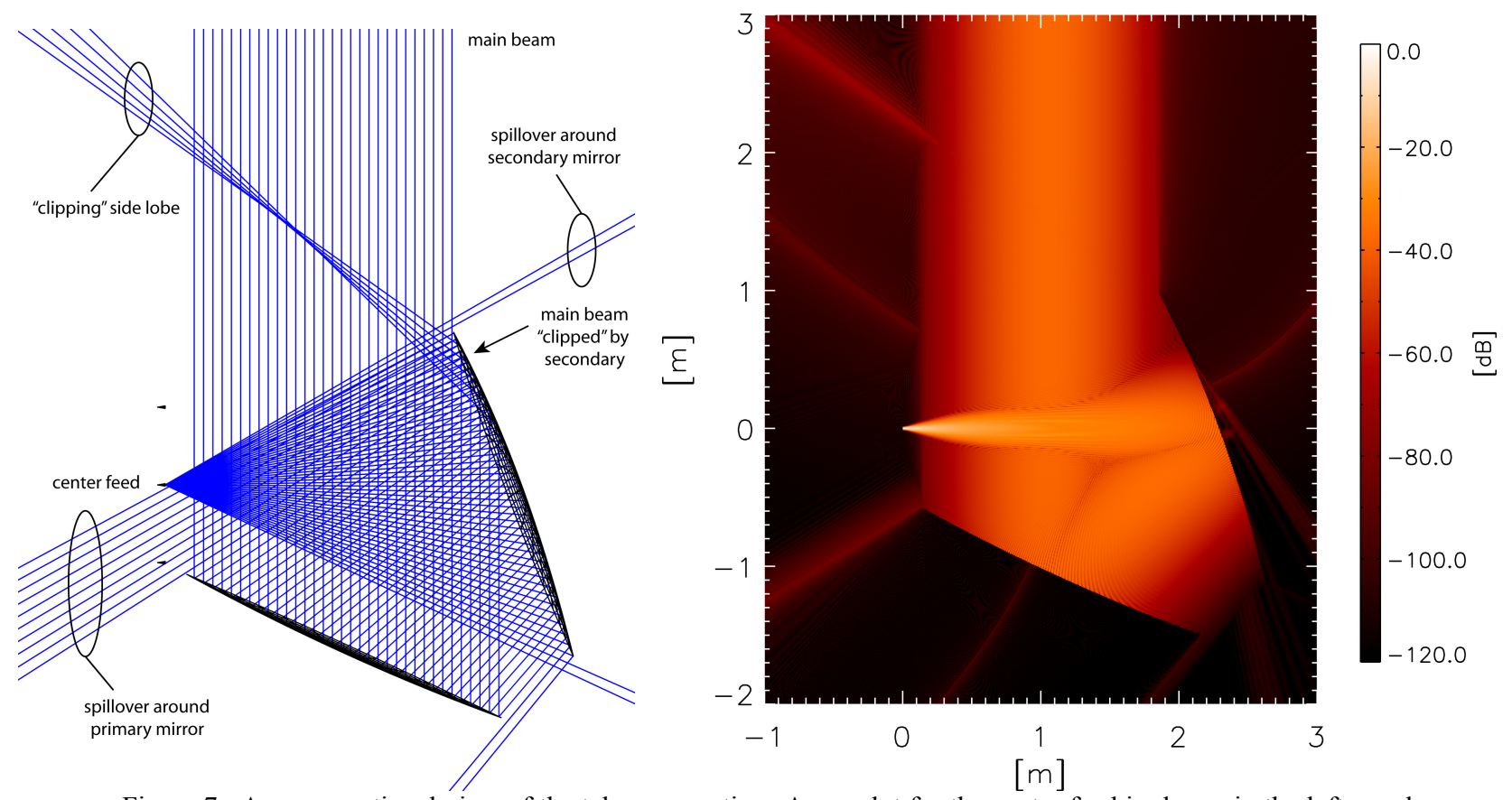

Figure 7. A cross-sectional view of the telescope optics. A ray plot for the center feed is shown in the left panel. A small fraction of the rays propagating from the primary mirror to the sky in the reverse time sense are "clipped" by the secondary mirror. These clipped rays produce a far side lobe. A physical optics representation of this effect is shown in the right panel. Here, the magnitude of the Poynting vector is plotted, and the signal is normalized to the maximum signal in the plot. The clipping side lobe is clearly visible in the upper left corner of this panel.

The results of the physical optics simulations show that the telescope itself has an uncorrected raw performance that is within the systematic goals, with the exception of the gain mismatch. The gain mismatch is caused by one linear 
polarization being preferentially absorbed due to oblique reflection of a slightly resistive reflector surface. This mismatch in gain is masked by any difference in gain or efficiency of the bolometers, and must be calibrated during inflight measurements.
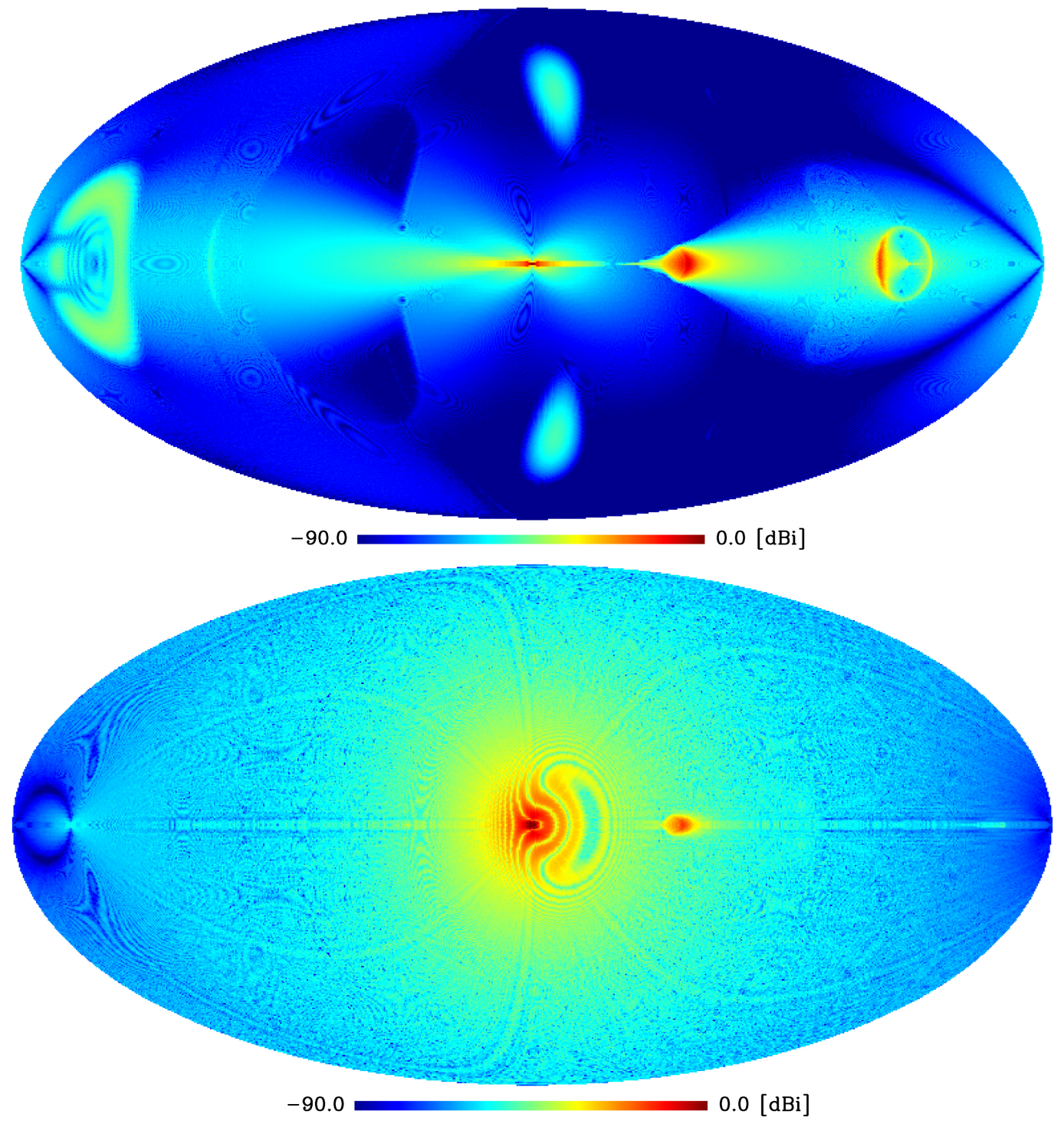

Figure 8. (top) A mollview projection of the all-sky telescope pattern assuming the telescope has no baffling. The main beam of the telescope is the bright dot in the center of the figure. The side lobes produce by spillover around the secondary and primary mirrors are the circular features at the left and right sides of the figure, respectively. The "clipping" side lobe appears just to the right of the main beam. The plot range was selected to highlight the far side lobes; the forward gain of the telescope is $65.1 \mathrm{dBi}$. The equatorial profile of this map is shown in Fig. 10. (bottom) The all-sky far side lobe pattern of the telescope with simulated baffling. For this computation, the effect of the telescope baffling was simulated using the aperture integration method. The equatorial profile of this map is shown in Fig. 9.

\section{FAR SIDELOBES}

A ray trace analysis of the telescope optics shows that the Crossed Dragone configuration should produce several different far side lobes. Example far side lobes can be seen in the cross-sectional view of the telescope in Fig. 7. While looking at the left panel in Fig. 7 and thinking in the reverse time sense, the far side lobes are correlated with any rays 
that originate from the "center feed" and terminate on the sky away from the main beam. Sky signals entering the telescope through these far side lobes can produce systematic errors in the observations that may be large compared to the sought-after gravity wave B-mode signal. The magnitude of any systematic error produced is related to the level of the associated far side lobes. It is not possible to determine these levels with ray plots. Therefore, to ascertain the overall performance of the telescope, we simulated a full-sky map of the telescope beam pattern using GRASP-9, which is a commercial physical optics software package.

\subsection{Physical Optics Analysis}

We computed the full-sky beam pattern of a hypothetical $150 \mathrm{GHz}$ feed located at the center of the focal plane of the telescope. The feed beam we used was linearly polarized and had an ideal Gaussian angular distribution. For the majority of the simulations, the taper of the feed beam was set to $-25 \mathrm{~dB}$ at a half angle of $13.13 \mathrm{deg}$. This feed taper corresponds to a horn with a $3.25 \mathrm{f} \lambda$ aperture diameter. Both polarization orientations of the feed beam were studied. The resulting far-side-lobe maps are shown in Fig. 8 and equatorial cuts of these maps are shown in Fig. 9.

We were particularly concerned with the "clipping" far side lobe highlighted in Fig. 7. Consequently, the physical optics simulations were designed to optimally study this effect. Simulations were done using two different methods. Both methods were computationally expensive with run times lasting up to approximately one month for one polarization of a single feed. The first method yielded the far-field beam pattern of the telescope assuming no telescope baffling. The second method simulated the effect of the telescope baffling by using the aperture integration method.

For both simulation methods, the feed was used as the radiation source. Surface currents driven by the fields emanating from the feed were computed on both the primary and secondary mirrors. These surface currents subsequently became radiation sources that ultimately radiated to the sky. To simulate the clipping effect, the currents computed on the secondary mirror were driven by two sources: the feed in the focal plane and radiation emanating from currents on the primary mirror. The number of points used to simulate the surface currents on the mirrors was set so that the far field beam patterns would be accurate to at least $-120 \mathrm{~dB}$. The map computed using the first method is shown in Fig. 8a. This map was computed using an $\mathrm{x}$-polarized feed. The $\mathrm{x}$ axis in the focal plane is parallel to the symmetry plane of the telescope.
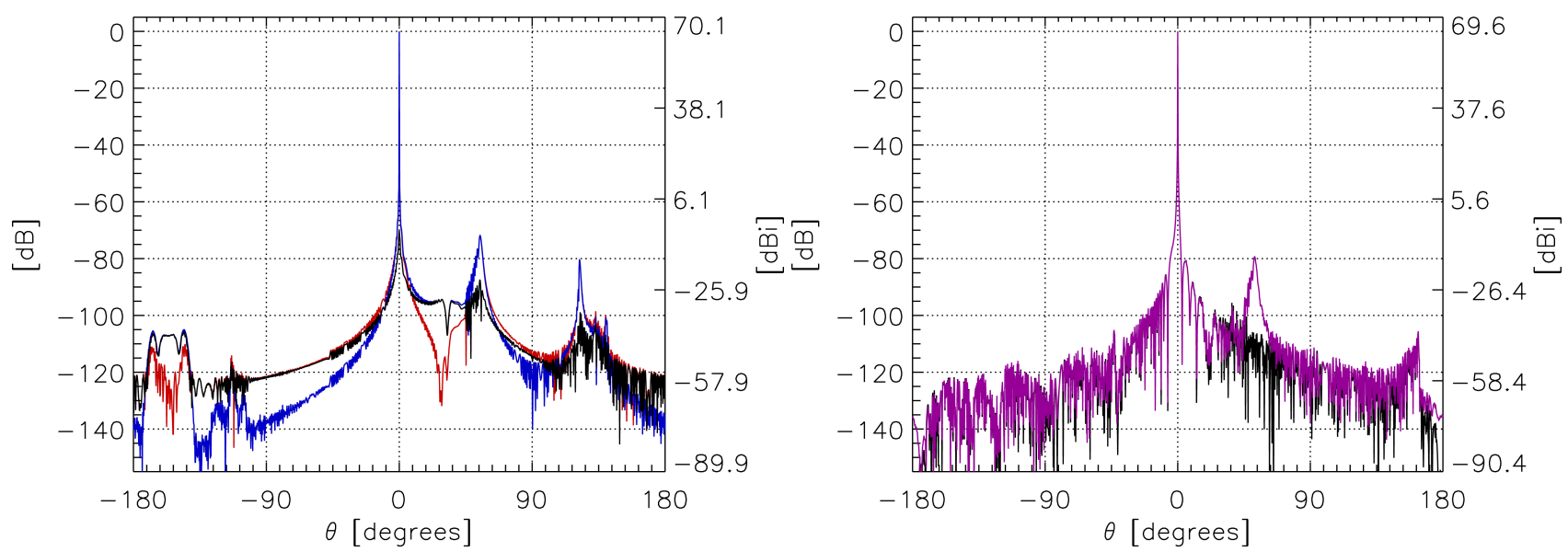

Figure 9. Telescope beam pattern profiles. The red curve plotted in the left panel is an equatorial cut of the map in Fig. 8a, which is the telescope beam pattern with no telescope baffling for an x-polarized feed. The simulated pattern for a y-polarized feed is plotted as the blue curve, while the difference between the two is the black curve. An equatorial cut of the map in Fig. $8 \mathrm{~b}$ is plotted as the purple curve in the right panel, which is the telescope beam pattern computed with the aperture integration method. Notice the far side lobes that appear near $\theta=-150$ $\operatorname{deg}$ and $\theta=120 \mathrm{deg}$ in the left panel do not appear in the right panel.

\subsection{Aperture Integration Method}

For the second method (the aperture integration method), the objective was to accurately calculate the stray light suppression of an absorbing baffle, and subsequently optimize the baffling design for maximum suppression. Suppose $\mathbf{J}$ 
and $\mathrm{M}$-- the electric and magnetic current sources -- radiate in presence of some scatterer inside a closed surface $\mathrm{S}$. The resultant field is denoted by $\boldsymbol{E}_{\boldsymbol{o}}, \boldsymbol{H}_{\boldsymbol{o}}$. The Equivalence Theorem states that in order to solve for the true fields outside, one can replace the field on the closed surface S, by a set of equivalent sources $\boldsymbol{J}_{\boldsymbol{e q}}, \boldsymbol{M}_{\boldsymbol{e q}}$. These equivalent sources can be chosen to make the field inside zero while giving rise to the exact field of $\left(\boldsymbol{E}_{\boldsymbol{o}}, \boldsymbol{H}_{\boldsymbol{o}}\right)$ outside S.

The aperture field integration method produces an excellent approximation to the Equivalence Theorem analysis, and was used for this calculation. Here, the aperture field was computed using physical optics and the physical theory of diffraction by including the following field contributions: radiation from the main reflector, radiation from the subreflector, direct radiation from the source feed, and secondary radiation from the subreflector, which results from the main reflector re-illuminating the subreflector (i.e the clipping side lobe). The aperture field was only calculated in the opening of the telescope baffle, and the field everywhere else was set to zero, since the baffle was modeled as a perfect absorber. The aperture field, consisting of both and $\mathrm{E}$ and $\mathrm{H}$ fields, was then radiated to the sky to produce the telescope beam pattern in Fig. 8b.

\subsection{GTD Analysis}

The EPIC-IM cold version has a pixel spacing of $2 \mathrm{f} \lambda$, implying roughly $15 \mathrm{~dB}$ higher edge taper than the $3.25 \mathrm{f} \lambda$ case. This adds significant computation time to far side lobe simulation. The geometric theory of diffraction (GTD) is a fast alternative to using physical optics to calculate the far side lobes, but it has the disadvantage that it potentially produces spurious artifacts.

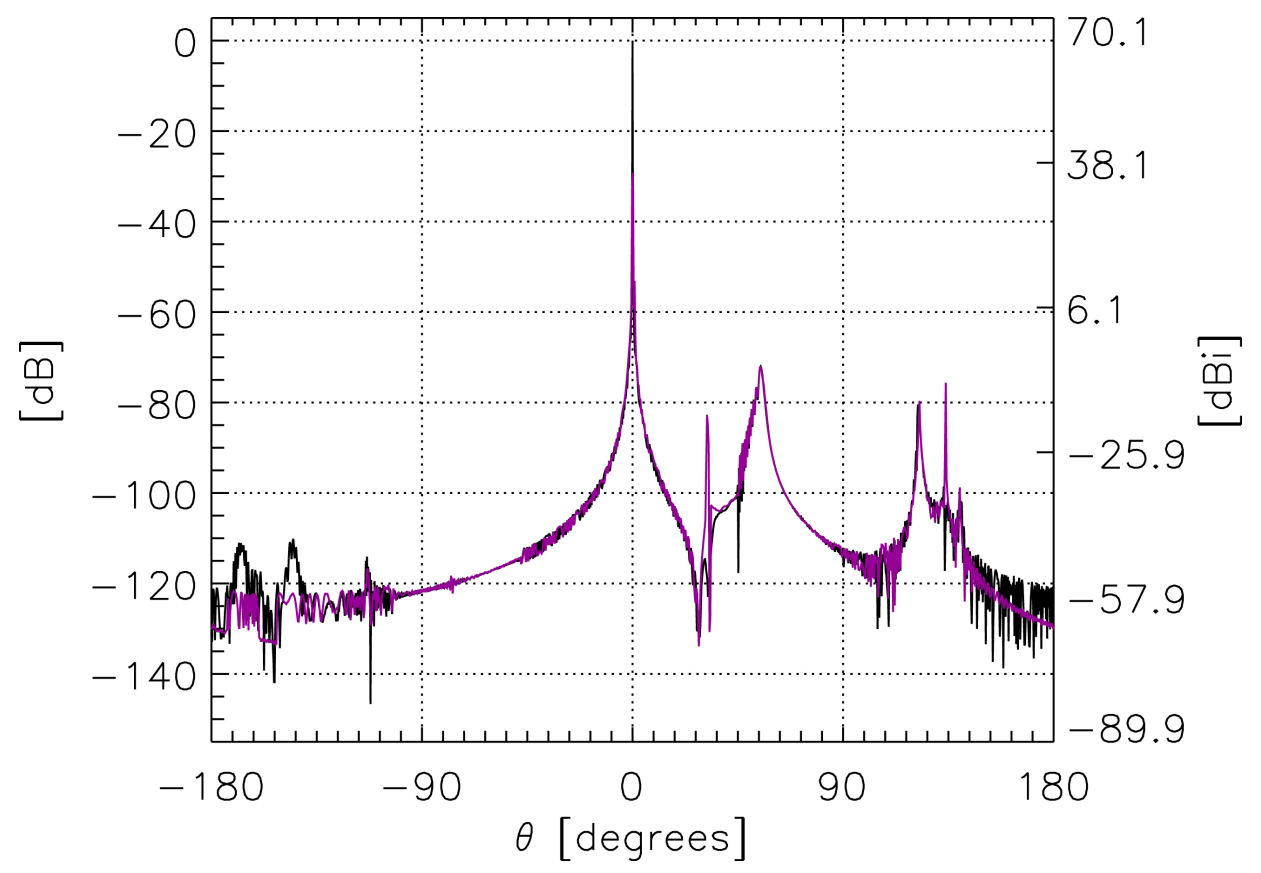

Figure 10. GTD vs PO+PTD for a $3.25 \mathrm{f} \lambda$ beam at the center of the EPIC-IM focal plane. The black curve is from a PO+PTD simulation, and the purple curve is from GTD. While the GTD results show some artifacts, the "clipping" side lobe at 60 degrees appears in both simulations.

The EPIC-IM 3.25 f $\lambda$ optics were analyzed by both GTD and PO+PTD with GRASP-9. GTD showed a reliable reproduction of the "clipping" side lobe, as shown in Fig. 10. This result provided enough confidence to analyze the 2 $\mathrm{f} \lambda$ case using the GTD. Fig. 11 is a plot of the GTD results for a $2 \mathrm{f} \lambda$ feed centered on the focal plane. For the purpose of this simulation, it is assumed that a $2 \mathrm{f} \lambda$ feed corresponds to a $-10 \mathrm{~dB}$ edge taper at the aperture, although the aperture itself is not included in this simulation. Both $\mathrm{x}$ and $\mathrm{y}$ linearly polarized beams were simulated for the central feed. The plots clearly show the "clipping" side lobe. The results additionally show that both polarizations are roughly equally sensitive to this side lobe, meaning that the side lobe is un-polarized. 


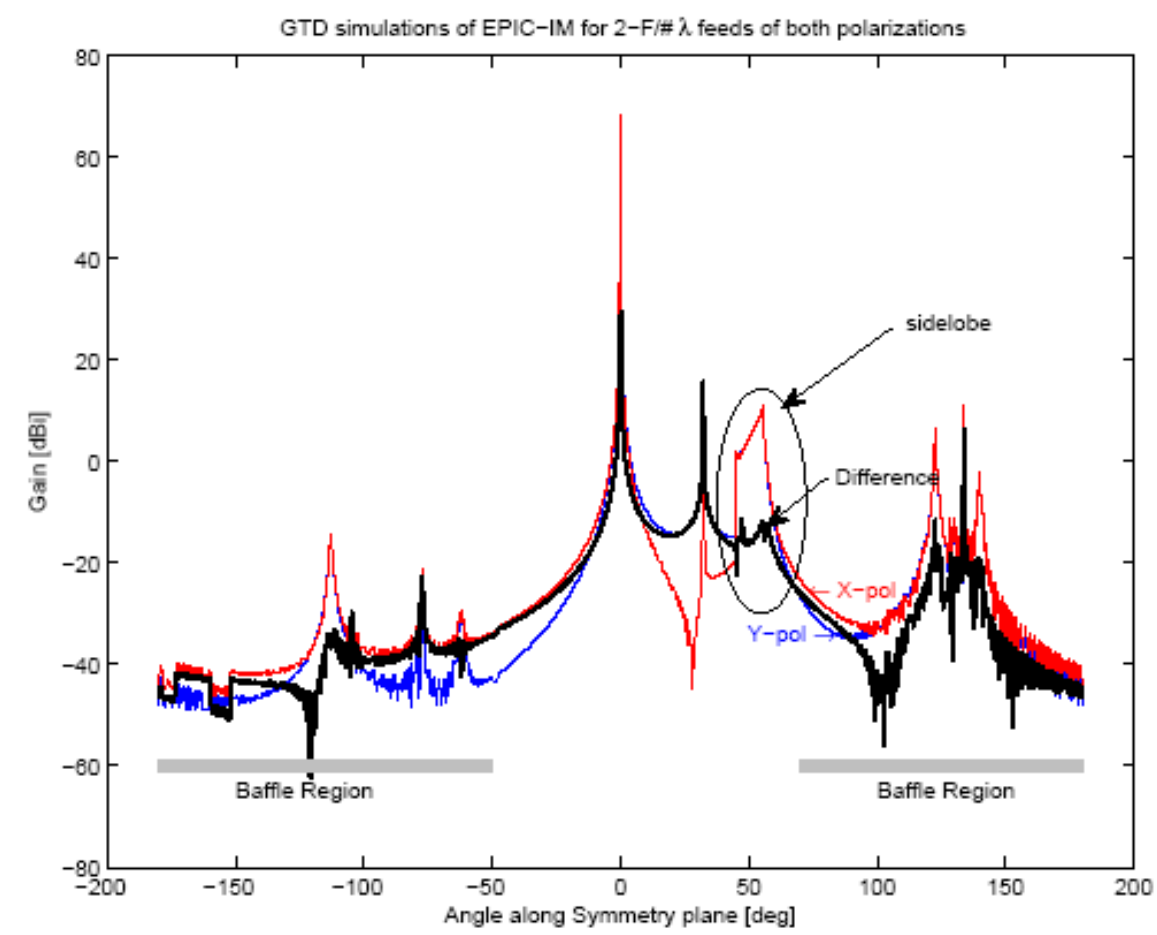

Figure 11. GTD simulation of the EPIC-IM (cold), 2 f $\lambda$ optics. The x-polarized results are shown in red, and the $y$ polarized in blue. The difference between the two polarization is shown in heavy black. The "clipping" side lobe is circled. From the difference, it is clear that the clipping side lobe is highly unpolarized. The feature at $+25 \mathrm{deg}$ does not appear in the more rigorous physical optics simulations, and is therefore likely an artifact. All other features will be absorbed by baffling, roughly denoted by the gray bars.

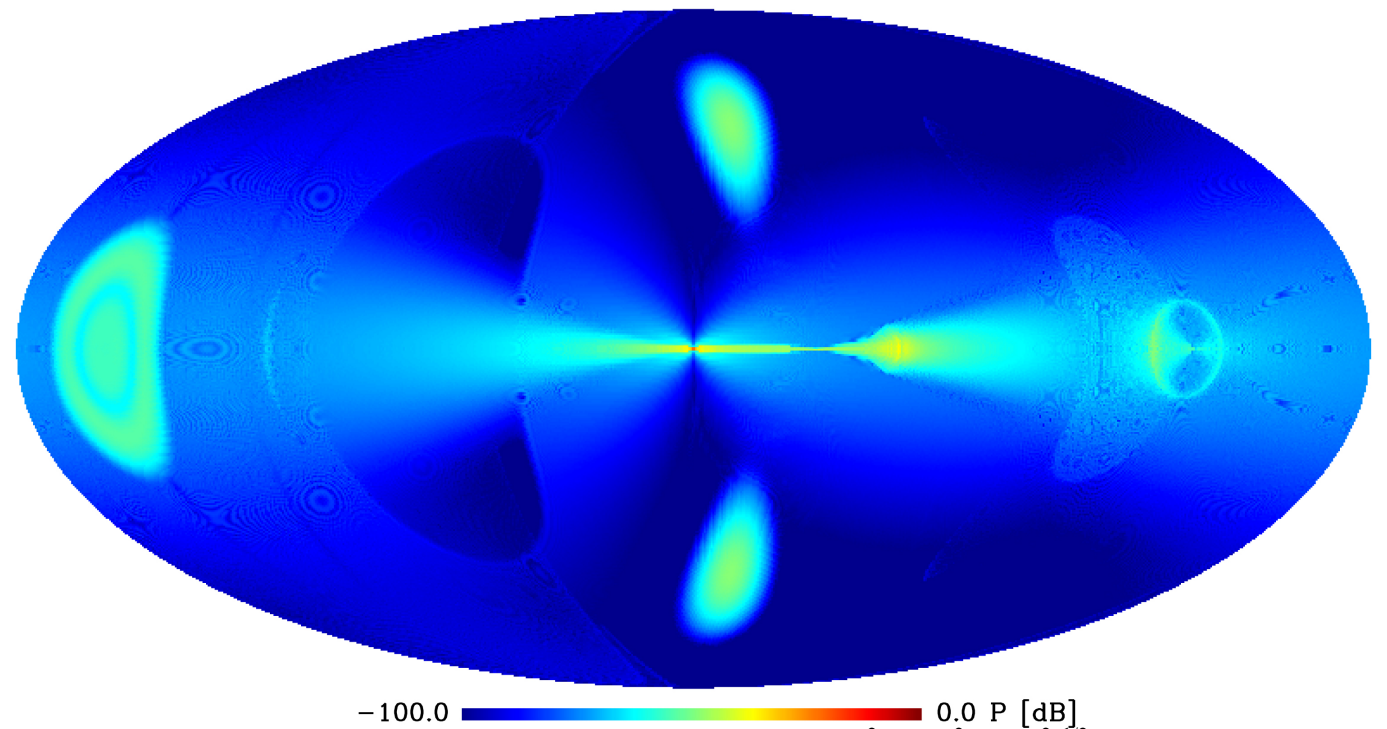

Figure 12. The polarization of the far side lobes. This map is the $\left(\mathrm{QT}^{2}+\mathrm{UT}^{2}+\mathrm{VT}^{2}\right)^{1 / 2}$. The forward gain of the TT beam is $54.1 \mathrm{~dB}$.

The $\mathrm{x}$ and $\mathrm{y}$-polarized far side lobe maps computed with method 1 were combined to produce Stokes parameter maps. The polarization map, which is defined here as $\left(\mathrm{QT}^{2}+\mathrm{UT}^{2}+\mathrm{VT}^{2}\right)^{1 / 2}$, is plotted in Fig. 11. This map indicates the level to which unpolarized sky signals become polarized. For reference, the maximum signal in the map is approximately 0 
$\mathrm{dB}$, and the forward gain of the associated TT map is $54.1 \mathrm{~dB}$. This information suggests the expected instrumental polarization is $10^{-3} \%$ for the center feed.

To determine whether this far side lobe performance is sufficient, the Stokes parameter maps were convolved with a map of the Galactic signals that are anticipated to appear at $150 \mathrm{GHz}$. For this convolution, the main beam of the telescope was suppressed using a mask. Therefore, the convolved sky signal map shows how the Galactic signals are redistributed via the far side lobes of the telescope. This redistributed signal is the systematic error we want to characterize. After the convolution, the magnitude of the spurious signal in each pixel was compared to the performance requirement, which assumes a tensor-to-scalar ratio of 0.001 .

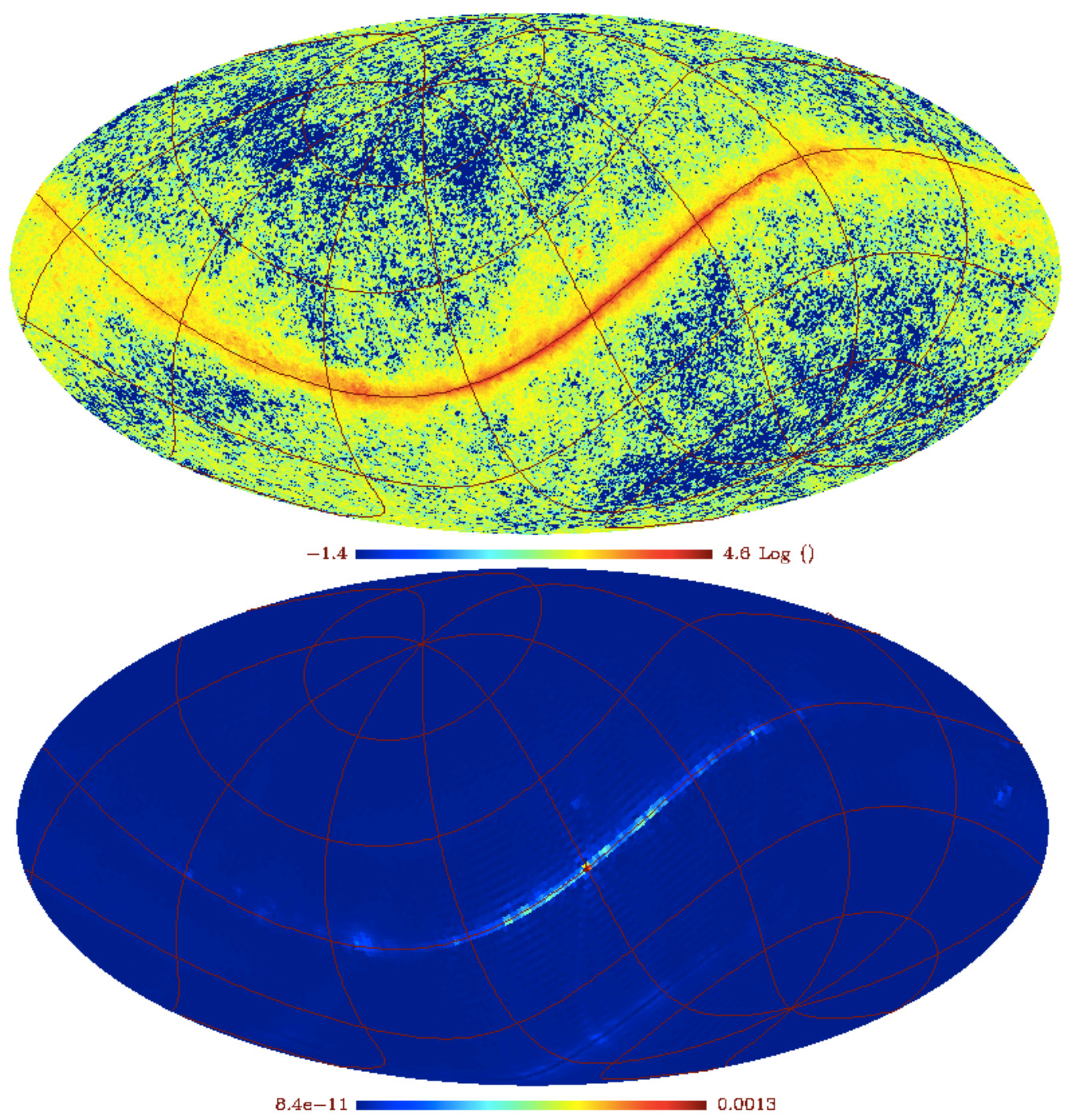

Figure 13. (top) The $150 \mathrm{GHz}$ sky map used for convolution with the far sidelobe pattern. Units are in $\log (\mathrm{mK})$. (bottom) The results of the convolution. Units are $\mu \mathrm{K}_{\mathrm{CMB}}$.

\section{GALACTIC SIGNAL FROM FAR SIDELOBES}

In order to evaluate the effect of the signal from the far sidelobes, we convolve the QT beam maps with a $150 \mathrm{GHz}$ sky model. The main beam is masked so only the contamination from the far sidelobes is calculated. Since the beam is asymmetric, it is necessary to rotate the beam with respect to the sky at each pointing to get the complete convolution. 
In order to further quantify this result, we make two histograms: the number of pixels with a given intensity, and the integrated histogram giving the total number of pixels less than a given intensity. This is similar to the plots that are shown for site surveys. By inspection one can see that fully $90 \%$ of the pixels are $<0.2 \mathrm{nK}$.
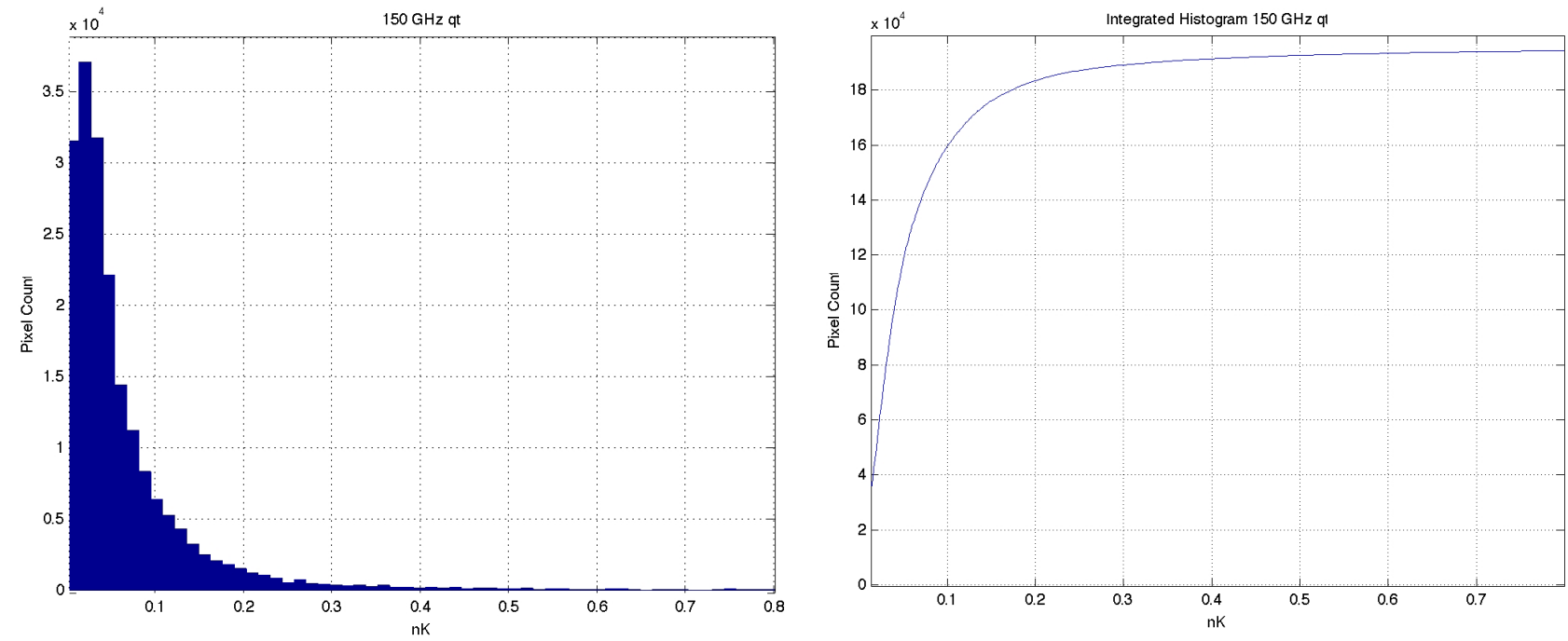

Figure 14. Histogram of signal values from Fig. 13b. The left panel is a histogram of leakages of galactic foregrounds into the EPIC-IM sidelobes. The right panel shows the same data as a cumulative distribution.

\section{SUMMARY AND FUTURE WORK}

The level of sidelobe control shown in Fig. 14 gives $\sim 0.1 \mathrm{nK}$ rms polarized signals over the majority of the sky, well within the required level of $3 \mathrm{nK}$, and the goal level of $1 \mathrm{nK}$, which are quoted for flat power spectra after band combination. However, this analysis was calculated in a single CMB band at $150 \mathrm{GHz}$ at the center of the focal plane assuming $3.25 \mathrm{f} \lambda$ illumination. These calculations must be extended to the $2 \mathrm{f} \lambda$ case. The scaling shown in Fig. 11 would indicate the level of far-sidelobes should be roughly at the required level of performance for the case of $2 \mathrm{f} \lambda$ illumination. Furthermore the calculation needs to be extended to cover the field of view and bands in the focal plane. Finally we note that we have not analyzed the use of optimized baffles, such as tapered absorbers surrounding the primary and aperture stop, or baffles to reduce the clipping lobe. These may offer further improvement in far-sidelobe performance.

\section{REFERENCES}

[1] Dodelson, E. et al., "The Origin of the Universe as Revealed Through the Polarization of the Cosmic Microwave Background", arXiv 0902.3796 (2009).

[2] Bock, J. et al., "Study of the Experimental Probe of Inflationary Cosmology (EPIC)-Intermediate Mission for NASA's Einstein Inflation Probe", arXiv 0906.1188 (2009).

[3] Bock, J. et al., "The Experimental Probe of Inflationary Cosmology (EPIC): A Mission Concept Study for NASA's Einstein Inflation Probe", arXiv 0805.4207 (2008).

[4] Weiss, R. et al., "Task Force on Cosmic Microwave Background Research", astro-ph 0605101 (2006).

[5] Chui, T., Bock, J., Holmes, W., and Raab, J., "Thermal Design and Analysis of a Multi-Stage $30 \mathrm{~K}$ radiative cooling system for EPIC", Cryogenics in press (2010).

[6] Shirron, P., "Cryogenic Technology for CMB-Pol: Sub-Kelvin Cooling”, Proc. Technology Development for a CMB Probe of Inflation, Boulder CO http://cmbpol.uchicago.edu/ (2008).

[7] Tran, H. and Page, L. "Optical Elements for a CMBPol Mission", Proc. Technology Development for a CMB Probe of Inflation, Boulder CO http://cmbpol.uchicago.edu/ (2008).

[8] Granet, C., "Designing classical dragonian offset dual-reflector antennas from combinations of prescribed geometric parameters", Antennas and Propagation Magazine, IEEE, 43(6):1045-9243 (2001). 